\title{
Management of patients eligible for proprotein convertase subtilisin/kexin type 9 (PCSK9) inhibitors: economic impact and reform proposals
}

Global \& Regional Health Technology

Assessment

Volume 2019: 1-12

(C) The Author(s) 2019

Article reuse guidelines: sagepub.com/journals-permissions DOI: | 0.1 | 77/22842403|986/375 journals.sagepub.com/home/grh @SAGE

\author{
Claudio Jommi ' 1 D and Marianna Cavazza ${ }^{2}$
}

\begin{abstract}
Background: PCSK9 inhibitors are indicated for primary hypercholesterolemia (heterozygous familial and non-familial) and for homozygous familial hypercholesterolemia (only evolocumab). They are subject to a drug registry for patient eligibility and follow-up. Patient access to PCSK9 inhibitors is still very limited and varies across regions.

Aim: This paper aimed to investigate the cost of the PCSK9-treated patient pathway and main barriers to patient access to medicines and to suggest some changes to this pathway.

Methods: The research was conducted through a literature review and an expert panel, which was surveyed through a Delphi approach. The panel included seven clinicians and five pharmacists.

Results: If patients are exempt from co-payment, the Italian National Health Service spends $€ 169.70$ and $€ 276.93$ per year to manage the PCSK9-treated patient pathway, depending on registry coverage (six or three treatment months, respectively). Non-exempt patients pay $€ \mid 76,43$ and $€ 27 I .53$ (for six and three months of coverage, respectively) per year. The referral process and the length of the pre-PCSK9 treatment period were considered the main barriers to access. The panelists suggested that the pre-PCSK9 treatment period be shortened to three months; they recommended six months of registry coverage, thereby decreasing the costs compared to three months' coverage; and they advised that outpatient specialists also be authorized to manage the follow-up. These actions may reduce pathway costs and enhance awareness of the disease.

Discussion: The study shows that the pathway of patients eligible for PCSK9 inhibitors can be improved. The surveyed experts integrated pathway-specific recommendations with broader advice to increase awareness on hypercholesterolemia in both citizens and general practitioners.
\end{abstract}

\section{Keywords}

PCSK9 inhibitors, patient pathway, costs, Delphi method

\section{Introduzione}

Alirocumab ed evolocumab sono indicati per ipercolesterolemia primaria (familiare eterozigote e non familiare) o da dislipidemia mista che non raggiungono livelli di LDL-C (low-density lipoprotein cholesterol) con la dose massima tollerata di una statina (i) in associazione a una statina o a una statina con altre terapie ipolipemizzanti, (ii) in monoterapia o in associazione ad altre terapie ipolipemizzanti in pazienti intolleranti alle statine o per $\mathrm{i}$ quali l'uso di statine è controindicato. Evolocumab è
'Professor of Government, Health and Not-for-Profit Organizations, CERGAS (Centre for Research on Health and Social Care

Management), SDA Bocconi, Milano, Italy

${ }^{2}$ Researcher, CERGAS (Centre for Research on Health and Social Care Management), SDA Bocconi, Milano, Italy

\section{Corresponding author:}

Claudio Jommi, Professor of Government, Health and Not-for-Profit Organizations, CERGAS (Centre for Research on Health and Social Care Management), SDA Bocconi, Via Bocconi 8, Milano, 20I3I, Italy.

Email: claudio.jommi@unibocconi.it 
indicato anche per ipercolesterolemia familiare omozigote, in associazione ad altre terapie ipolipemizzanti negli adulti e negli adolescenti di almeno 12 anni di età.

Gli inibitori di proproteina convertasi subtilisina/kexina di tipo 9 (PCSK9) sono soggetti a registro di monitoraggio che ne specifica ulteriormente le condizioni di rimborsabilità, prevista per pazienti di età $\leqslant 80$ anni:

- in prevenzione secondaria con ipercolesterolemia familiare eterozigote o ipercolesterolemia non familiare o dislipidemia mista con livelli di LDL-C $\geqslant 100 \mathrm{mg} / \mathrm{dL}$, nonostante terapia da almeno sei mesi con statina ad alta potenza alla massima dose tollerata (atorvastatina $40-80 \mathrm{mg}$ oppure rosuvastatina $20-40$ $\mathrm{mg})+$ ezetimibe oppure con dimostrata intolleranza alle statine da parte dei pazienti;

- in prevenzione primaria con ipercolesterolemia familiare eterozigote e livelli di LDL-C $\geqslant 130 \mathrm{mg} / \mathrm{dL}$ nonostante terapia da almeno sei mesi con statina ad alta potenza alla massima dose tollerata (atorvastatina $40-80 \mathrm{mg}$ oppure rosuvastatina 20 $40 \mathrm{mg})+$ ezetimibe o in pazienti con dimostrata intolleranza alle statine.

Il registro specifica anche la numerosità e la frequenza delle determinazioni lipidiche (tre determinazioni, eseguite in momenti diversi, del profilo lipidico con intervallo tra una determinazione e l'altra di almeno due mesi) e i criteri per la valutazione dell'intolleranza alle statine.

I farmaci sono soggetti a prescrizione medica limitativa, dispensabili al pubblico su prescrizione di centri ospedalieri e di centri specialistici individuati dalle regioni e su prescrizione di cardiologo e internista. Il farmaco è inserito nel Prontuario della Distribuzione Diretta (PHT): si tratta dell'unico caso di farmaci non a designazione orfana e per patologie croniche per i quali sono previsti contemporaneamente registro e PHT.

$\mathrm{La}$ prescrizione a carico del Servizio Sanitario Nazionale (SSN) prevede la compilazione, attraverso registro web-based, di una scheda "eleggibilità e dati clinici" (EDC), una scheda di richiesta del farmaco, una scheda di follow-up (rivalutazione) ogni tre/sei mesi, con conseguente accesso del paziente all'ospedale quattro/due volte all'anno e un'eventuale scheda di fine trattamento. Tutto il percorso terapeutico (prima prescrizione e prescrizioni successive in follow-up) e la dispensazione del farmaco avvengono in ambiente ospedaliero.

Oltre alle schede compilate dal medico prescrittore è prevista la compilazione di una scheda di dispensazione da parte del farmacista ospedaliero, con frequenza tendenzialmente pari a tre mesi, in linea con i tempi di rendicontazione e scarico del farmaco su File $\mathrm{F}$.

L'accordo di accesso negoziato stipulato tra l'Agenzia Italiana del Farmaco (AIFA) e le due imprese titolari dell'Autorizzazione all'Immissione in Commercio (AIC)

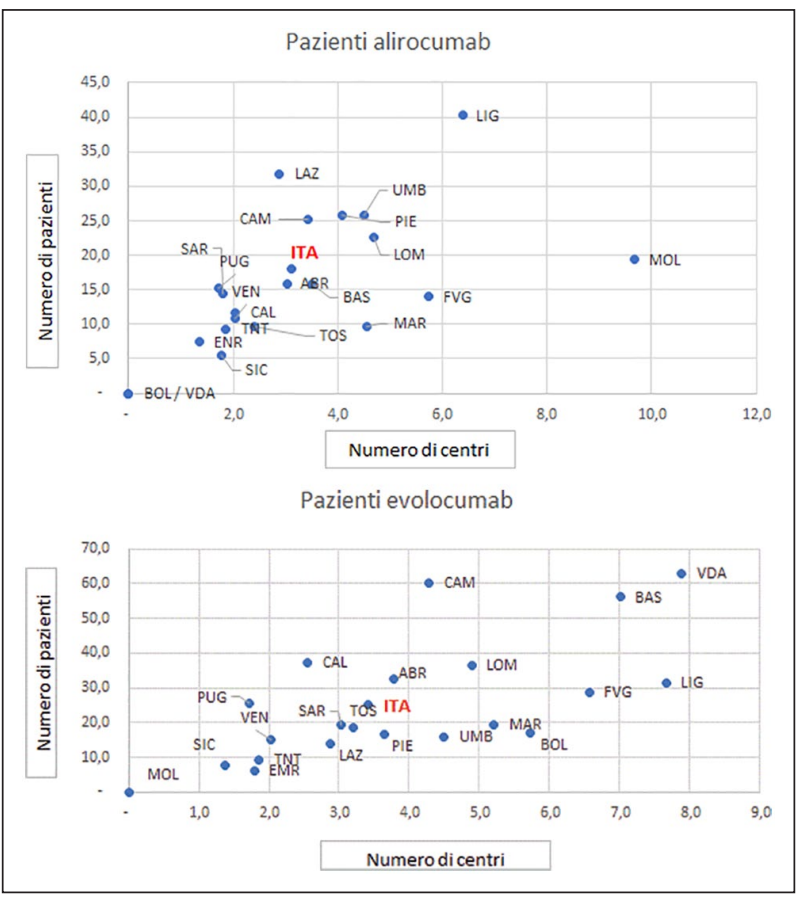

Figura I. Numero di centri per milione di abitanti e numero di pazienti per milione di abitanti.

Fonte: nostra elaborazione su dati Rapporto OsMed 2018.

ABR: Abruzzo; BAS: Basilicata; CAL: Calabria; CAM: Campania; EMR: Emilia Romagna; FVG: Friuli Venezia Giulia: LAZ: Lazio; LIG: Liguria; LOM: Lombardia; MAR: Marche: MOL: Molise; PIE: Piemonte; BOL: Provincia Autonoma di Bolzano; TNT: Provincia Autonomia di Trento; PUG: Puglia; SAR: Sardegna; SIC: Sicilia; TOS: Toscana; UMB: Umbria; VDA: Valle d'Aosta; VEN: Veneto; ITA: Italia.

contempla uno sconto progressivo in base al meccanismo prezzo/volume.

Sono attualmente poche le evidenze di letteratura sull'uso di tali farmaci nella pratica clinica in Italia. Secondo i dati dell'ultimo rapporto dall'Osservatorio sull'Impiego dei Medicinali (Rapporto OsMed), ${ }^{1}$ i pazienti trattati con evolocumab e alirocumab sarebbero stati 2.608 nel 2017. Sulla base di estrapolazione su dati epidemiologici della Regione Veneto, ${ }^{2}$ i pazienti eleggibili al trattamento sarebbero pari a 19.000 in Italia. Il livello di copertura della popolazione target era quindi nel 2017 ancora molto basso (13,7\% dei pazienti eleggibili).

Dai dati del Rapporto OsMed emerge anche una notevole variabilità nel livello di accesso, non giustificata dal quadro epidemiologico, e una correlazione tra centri abilitati e pazienti in trattamento nel 2017 (Figura 1).

Il dato 2017 è però condizionato dalla gradualità con cui le regioni hanno incluso il farmaco nei prontuari regionali e dai tempi di identificazione dei centri specialistici da parte delle regioni. Con riferimento al secondo aspetto, è interessante rilevare il fatto che alcune Regioni (Lombardia, Puglia e Veneto) hanno identificato i centri abilitati tra febbraio e marzo 2017,3 ovvero immediatamente dopo la Determina AIFA di Prezzo e 
Rimborso (1 febbraio per evolocumab ${ }^{4}$ e 16 febbraio 2017 per alirocumab ${ }^{5}$ ), mentre altre regioni hanno operato successivamente (ad esempio, Lazio a luglio 2017 ed Emilia Romagna a novembre 2017). ${ }^{3}$

Un secondo dato interessante, tratto dal Rapporto OsMed, è l'incidenza di avvio alla terapia per intolleranze al trattamento con statine, di poco inferiore al $50 \%$ nel nostro Paese, che sembra essere superiore alle casistiche internazionali. L'elevata incidenza di pazienti statinointolleranti può significare un arruolamento preferenziale di pazienti a elevato rischio, senza opzioni terapeutiche disponibili efficaci perché intolleranti.

È quindi evidente che l'accesso ai farmaci inibitori di PCSK9 (PCSK9-inibitori) è ancora inferiore al livello atteso e che il sistema di gestione del percorso terapeutico del paziente (eleggibilità, trattamento e follow-up), per quanto ispirato dall'obiettivo di rendere appropriato l'uso di tali farmaci, presenta elementi di complessità che ne hanno rallentato l'accesso.

A questo si aggiunge una certa variabilità del profilo di costo-efficacia di tali farmaci influenzata anche dal contesto sanitario di riferimento dell'analisi (il National Institute for Health and Care Excellence (NICE) li ha raccomandati ma con sconto obbligatorio). ${ }^{6-8}$

\section{Obiettivo della ricerca}

Il primo obiettivo dello studio è analizzare l'attuale gestione del percorso terapeutico del paziente potenzialmente eleggibile al trattamento con farmaci PCSK9-inibitori, rilevandone il carico amministrativo e il costo nella prospettiva del sistema sanitario (prestazioni a carico del SSN e dei pazienti). Il secondo obiettivo è verificare, con il supporto di un panel di esperti clinici e farmacisti operanti nei servizi farmaceutici regionali e nelle aziende sanitarie, le possibili opzioni di una modifica di tale percorso. Tali opzioni sarebbero finalizzate, tra gli altri aspetti, a ridurre il costo del percorso, mantenendone l'obiettivo finale, rappresentato dall'appropriatezza prescrittiva del farmaco e dall'adeguatezza del follow-up.

\section{Metodo della ricerca}

La ricerca è stata svolta sulla base delle evidenze esistenti sul percorso attuale e su una valutazione percettiva circa le principali criticità di tale percorso e l'opportunità di modificarlo da parte di un panel di esperti.

Più nello specifico, ai rispondenti è stato attribuito un ruolo (i) informativo, ovvero finalizzato a integrare / valutare criticamente le informazioni raccolte per la validazione e la stima del costo di gestione del percorso terapeutico attuale, (ii) valutativo, ovvero finalizzato a esprimere un'opinione su opportunità e priorità di alcune proposte di modifica di tale percorso.
Come sopra specificato, il costo della gestione del percorso è stato valutato nella prospettiva del sistema sanitario e comprende l'erogazione di prestazioni sanitarie (visite e prestazioni di laboratorio) e la compilazione delle schede di registro.

La definizione di priorità è avvenuta su una scala di preferenza. Il grado di concordanza è stato valutato sulla base dell'indice W di Kendall, indice compreso tra $0 \%$ (concordanza nulla) e 100\% (concordanza assoluta). L'indice $\mathrm{W}$ di Kendall è calcolato come:

$$
\begin{gathered}
\mathrm{W}=12 / \mathrm{Sm}^{2} \mathrm{n}\left(\mathrm{n}^{2}-1\right) \\
\text { dove } \mathrm{S}=\sum_{j=1}^{m}(R-\bar{R})^{2}
\end{gathered}
$$

dove (n) è il numero dei rispondenti, (R) è il rango attribuito all'oggetto $(\mathrm{m})$ e (R) è il valore medio del rango j-esimo, (S) è la varianza campionaria dei ranghi e il termine 12/ $\mathrm{m}^{2} \mathrm{n}\left(\mathrm{n}^{2}-1\right)$ è una costante di normalizzazione. ${ }^{9}$

$\mathrm{La}$ valutazione di accordo sulle proposte di cambiamento del percorso è avvenuta sulla base di una scala di Likert a quattro dimensioni, per evitare un posizionamento centrale dei rispondenti, possibile su scale dispari. ${ }^{10}$ Ai quattro livelli di accordo è stato attribuito un punteggio pari a 1 (per nulla d'accordo), 2 (poco), 3 (abbastanza), 4 (molto) e sono stati calcolati valore medio e coefficiente di variazione $(\mathrm{CV})$ delle risposte. Il CV, pari al rapporto tra deviazione standard e media, è un indicatore di dispersione rispetto alla media, indipendente dall'unità di misura della variabile.

La raccolta di informazione dai rispondenti è avvenuta attraverso il metodo Delphi. La metodologia Delphi prevede l'interlocuzione tra ricercatore e rispondenti, che operano in cieco tra di loro, attraverso passi successivi (massimo tre) finalizzati a trovare il consenso su una rappresentazione finale delle valutazioni. ${ }^{11} \mathrm{La}$ tecnica Delphi ha il vantaggio di consentire un contributo "paritario" da parte degli esperti, essendo il parere espresso in cieco, con successivo confronto / mediazione da parte del ricercatore nei passi successivi al primo. La scelta dei rispondenti si è basata (i) sulla rappresentatività delle differenti professioni coinvolte (clinici e farmacisti) e delle diverse realtà regionali; (ii) sulla circostanza che i rispondenti fossero attivi sul fronte delle politiche regionali / locali implementate per la gestione dei farmaci e il miglioramento della prevenzione in ambito cardiovascolare. La selezione degli esperti è avvenuta sulla base dell'interesse da parte dei candidati a partecipare al progetto di ricerca (sample of convenience). ${ }^{12}$

Nello specifico, le fasi del Delphi sono state le seguenti:

- invio a dicembre 2018 da parte dei ricercatori di un documento base su cui i rispondenti hanno lavorato a distanza in cieco secondo metodica Delphi; 


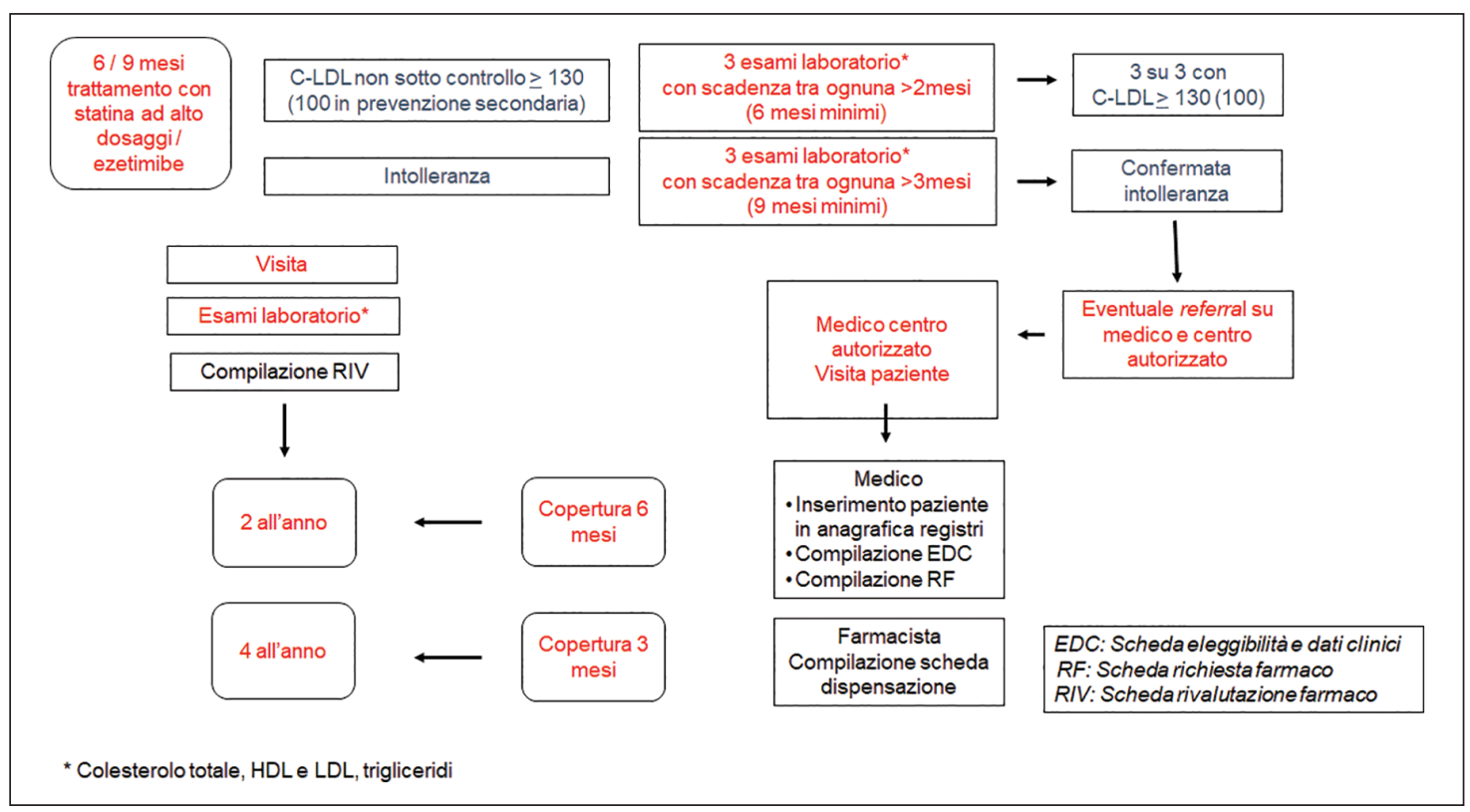

Figura 2. La gestione del percorso del paziente candidabile ai PCSK9-inibitori.

Nota: In rosso sono indicate le prestazioni tariffate, in nero quelle svolte da clinici e farmacisti. Il percorso è descritto sulla base di quanto specificato nelle schede AIFA (www.cardiotool.net).

- due round di revisione del documento tra gennaio e febbraio;

- validazione e discussione finale del documento nell'ambito di un incontro a fine febbraio.

Sono stati coinvolti 12 esperti (sette clinici e cinque farmacisti di sette diverse regioni - Piemonte, Lombardia, Friuli VG, Toscana, Lazio, Campania, Sicilia). Non tutti i rispondenti hanno risposto. Al primo round hanno risposto 10 rispondenti (sei clinici e quattro farmacisti). Le analisi sono state effettuate sui 10 rispondenti iniziali. L'undicesimo (clinico) e il dodicesimo (farmacista) sono intervenuti a completamento dell'analisi sotto il profilo qualitativo, validando di fatto la sintesi delle valutazioni effettuate dai colleghi. I rispondenti sono tutti citati nella sezione "Ringraziamenti" e hanno approvato il presente testo.

\section{I risultati: la gestione attuale del percorso terapeutico}

La gestione teorica del percorso del paziente eleggibile al trattamento con PCSK9-inibitori in prevenzione primaria e secondaria è rappresentata dalla Figura 2. Per i pazienti con ipercolesterolemia familiare omozigote (solo evolocumab) non è prevista la finestra temporale antecedente alla compilazione della scheda di arruolamento.

Secondo gran parte dei rispondenti, la Figura 2 rappresenta il percorso reale del paziente nella propria regione. Con riferimento alla copertura del registro il $70 \%$ dei rispondenti ha specificato "sei mesi" mentre il 30\% "tre mesi". Con riferimento invece alla durata della copertura della terapia, il $70 \%$ dei rispondenti ha risposto "tre mesi" e il 30\% invece un mese. La Figura 2 rappresenta, quindi, il percorso effettivamente realizzato, con approcci più intensivi in alcuni ambiti (rivalutazione clinica quattro volte all'anno) e accessi per la dispensazione del farmaco anche di 12 volte all'anno (copertura della terapia di un mese).

I farmaci PCSK9-inibitori vengono dispensati dalla farmacia dell'ospedale in cui il paziente viene visitato. Tre rispondenti hanno segnalato che il farmaco è dispensato dalla farmacia della azienda sanitaria locale di residenza del paziente. Laddove è stato definito un centro / ambulatorio territoriale di riferimento, la dispensazione del farmaco avviene da parte della farmacia territoriale dell'azienda sanitaria attraverso il distretto di appartenenza dell'assistito sia per le prescrizioni che provengono dai centri ospedalieri $(40 \%)$ sia per le prescrizioni originate dal centro territoriale $(60 \%)$ della stessa azienda.

Con riferimento invece alla prescrizione dei PCSK9inibitori, sono state rilevate delle iniziative di controllo da parte di alcuni rispondenti. Si tratta (i) della previsione di un'attestazione specifica sull'aderenza al trattamento con statina ed ezetimibe per la verifica di eleggibilità al trattamento con PCSK9-inibitori, (ii) di azioni sistematiche di monitoraggio delle prescrizioni, (iii) della possibilità per le farmacie ospedaliere di definire dei tetti di spesa dedicati (e non nell'ambito di eventuali più ampi tetti/target di spesa per farmaci rimborsati attraverso il File F).

La Regione Friuli Venezia Giulia ha segnalato come, a partire dal 2019, (i) venga effettuato, su scelta volontaria delle aziende sanitarie, un approfondimento 
sull'utilizzo dei PCSK9-inibitori, per una valutazione sull'appropriatezza prescrittiva, sulla tipologia di pazienti trattati e sui risultati raggiunti in termini di riduzione del valore di LDL-C; (ii) venga previsto il coinvolgimento in tale iniziativa del centro prescrittore (cardiologia) e del servizio farmaceutico aziendale.

Il costo della gestione del percorso nella prospettiva del sistema sanitario comprende quello dell'erogazione di prestazioni sanitarie (visite e prestazioni di laboratorio) e quello della compilazione delle schede di registro.

La Tabella 1 illustra il valore delle prestazioni tariffate collegate alla fase di verifica delle condizioni di eleggibilità e alla rivalutazione.

$\mathrm{Si}$ osserva che il valore tariffario non coincide necessariamente con il costo delle prestazioni erogate e che per le prestazioni ambulatoriali è prevista una compartecipazione massima a carico del paziente (non esente) di $€ 36,15$ per prestazione (tutte le prestazioni previste nella gestione del percorso hanno tariffe più basse) e una quota fissa di $€ 10$ (il cosiddetto superticket, confermato anche per il 2019). Nello specifico, è prevista un'applicazione del superticket:

- pieno in Liguria, Friuli Venezia Giulia, Lazio, Marche, Molise, Puglia, Calabria, Sicilia, Sardegna;

- in base al reddito in Veneto, Emilia-Romagna, Toscana, Umbria, Abruzzo, Campania;

- in base al costo della prestazione erogata in Piemonte e Lombardia;

- pari a $€ 3$ nella Provincia Autonoma di Trento;

- pieno per ricette superiori ai $€ 20$ in Valle d'Aosta.

Solo la Provincia Autonoma di Bolzano e la Basilicata non hanno introdotto il superticket. ${ }^{14}$

Le prestazioni collegate al percorso sono formalmente coperte dal SSN, ma sono previste compartecipazioni che di fatto, dato il valore delle tariffe, pongono la prestazione a carico del paziente, a meno che lo stesso paziente non sia esente. L'esenzione da compartecipazione è prevista, nello specifico, dal codice esenzione 025 che include i pazienti con ipercolesterolemia familiare eterozigote IIa e IIb, primitiva poligenica, familiare combinata $\mathrm{e}$ iperlipoproteinemia, e dal codice RCG070, che include ipercolesterolemia familiare omozigote IIa e IIb. ${ }^{15}$

Il valore complessivo delle prestazioni tariffate (a tariffe nazionali) è compreso tra $€ 151,53$ e $€ 96,43$ nel caso, rispettivamente, di copertura a tre e sei mesi. Tale valore tariffario scenderebbe a $€ 68,88$ con una copertura di un anno. In caso pagamento di un superticket a prestazione, il costo a carico del paziente sale a $€ 176,43$ e $€ 271,53$ nel caso, rispettivamente, di copertura a sei e a tre mesi ( $€ 128,88$ con una copertura di un anno) (Tabella 1$)$.

Al valore tariffario delle prestazioni si aggiungono i costi collegati alla compilazione delle schede previste dal registro AIFA, costi a carico del SSN. I rispondenti hanno

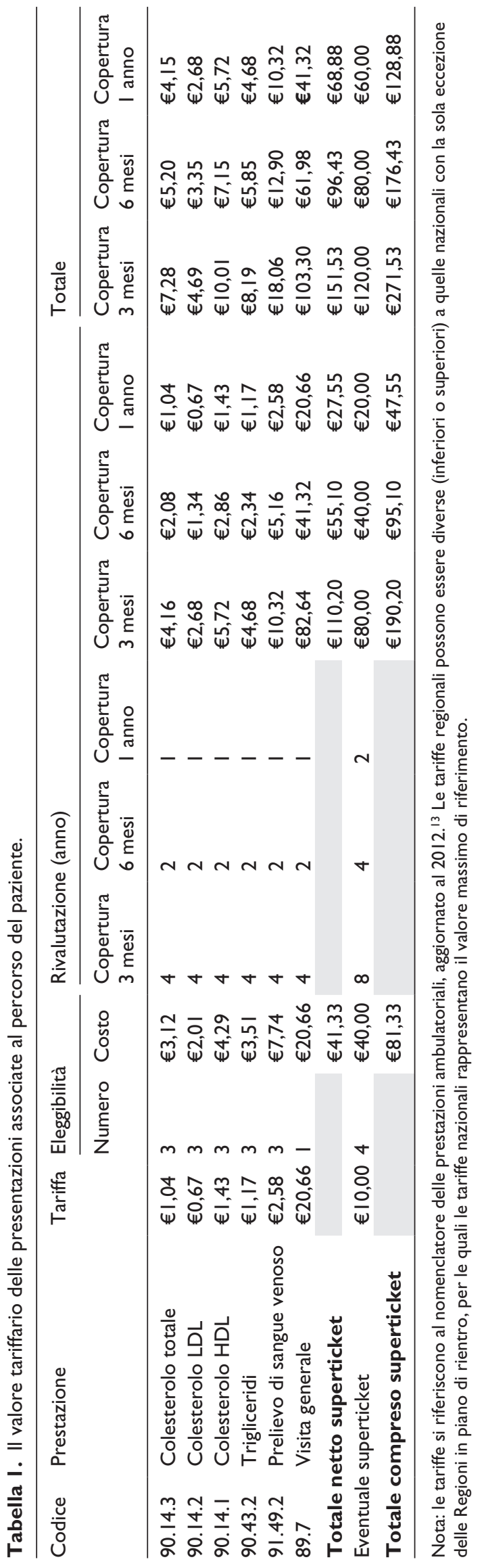


dato indicazioni sui tempi dedicati dai clinici e farmacisti alla compilazione delle schede.

Nello specifico il tempo medio dedicato alla:

- "Scheda eleggibilità e dati clinici" è di 20 minuti (min $10-\max 30$ );

- "Scheda richiesta farmaco" è di 10 minuti (min 3 $\max 15)$;

- "Scheda rivalutazione pazienti" è di 10 minuti (min $5-\max 15)$

- "Scheda dispensazione farmaco" è di 7 minuti (min 3 - $\max 15)$.

Gli stessi rispondenti hanno evidenziato come, oltre al tempo dedicato dai clinici e farmacisti per la compilazione delle schede, esiste un'attività di supporto, fornita dal personale infermieristico e amministrativo, difficilmente stimabile e che in questa sede non è stata valorizzata sotto il profilo economico.

I rispondenti hanno evidenziato come il tempo di compilazione delle schede (soprattutto di eleggibilità) dipenda molto dal tipo di paziente e dal referral del medico sul territorio e tenda a decrescere nel tempo per effetto della maturata esperienza.

I tempi sono stati convertiti poi in costo complessivo sulla base di una stima del costo unitario lordo del personale medico e farmacista. Il costo medio lordo annuo di un farmacista ospedaliero è stato stimato come media delle retribuzioni tabellari e delle indennità previste, ${ }^{16}$ quello dei medici (ospedalieri) è stato desunto dal Rapporto ARANsulle RetribuzioninellaPubblicaAmministrazione. ${ }^{17}$ Entrambi i dati si riferiscono al 2016: dato il mancato rinnovo dei contratti, non è stato effettuato alcun aggiustamento sulla base del tasso di inflazione. Il costo medio lordo orario è stato stimato rapportando lo stipendio lordo mensile al numero di ore settimanali (36) moltiplicate per le settimane lavorative (46). In totale tali costi sono pari a $€ 52,33$ e $€ 89,57$ nel caso, rispettivamente, di copertura a sei e a tre mesi da parte del registro.

Per valutare i costi pieni di compilazione delle schede è necessario aggiungere ai costi diretti del personale gli altri costi diretti (beni e servizi e quote ammortamento direttamente attribuibili), i costi dei servizi indirettamente attribuibili su base specifica e i costi generali attribuibili su base generica. Non essendo stati raccolti tali dati nelle sedi aziendali interessate e non essendovi dati robusti su quale quota rappresentino i costi indiretti e generali sul totale dei costi delle aziende sanitarie, si è effettuata una stima a partire dai dati contabili 2017. ${ }^{14}$ In media, secondo tali dati, il personale dipendente rappresenta il $29,2 \%$ dei costi complessivi nelle aziende sanitarie, mentre i beni e servizi (non gestiti in economia) rappresentano il $34,7 \%$ e gli ammortamenti il $2 \%$, che, aggiunti ai beni e servizi sopra menzionati danno un totale del $36,7 \%$. Gli altri costi sono servizi sanitari erogati in regime di accreditamento / convenzione che non sono oggetto di ribaltamento sull'attività di compilazione del registro. Poiché tale attività è a uso intensivo di lavoro, si è ipotizzato che circa un terzo dei costi dei beni e servizi e quote di ammortamento $(12,2 \%$ dei costi totali) possa essere associato a costi diretti (non stimati in questa sede) e a costi ribaltabili su un tale tipo di attività, il che equivale ad aumentare del $40 \%$ il costo del personale. Se ai costi del personale si aggiungono gli altri costi diretti non stimati in questa sede e quelli indiretti / generali ribaltati pro quota, si ottiene un costo compreso tra $€ 73,27$ e $€ 125,40$ ( $€ 47,20$ in caso di copertura di un anno) (Tabella 2).

La Tabella 3 analizza i costi complessivi nello scenario di copertura del registro di tre mesi, sei mesi (e un anno) al lordo e al netto del superticket, laddove applicato.

\section{Risultati: possibili modifiche del percorso}

Un recente contributo ${ }^{18}$ ha discusso le principali problematiche nell'accesso ai farmaci PCSK9-inibitori, sottolineando in particolare (i) la complessità nella compilazione del registro; (ii) la copertura limitata del registro (tre/sei mesi) che comporta un frequente accesso agli ambulatori specialistici ospedalieri da parte dei pazienti per il rinnovo, poco compatibili con la normale sequenza di visite di controllo in Italia. Lo studio riferisce infatti come a un paziente acuto in ambito cardiovascolare si consigli un primo controllo dopo quattro-sei settimane dall'evento e i successivi sulla base delle esigenze cliniche del paziente; (iii) il trattamento con statine ad alto dosaggio ed ezetimibe per almeno sei mesi, con riduzione delle potenzialità di accesso in termini generali; (iv) la prescrivibilità riservata a poche figure cliniche, indicando come altri possibili specialisti il neurologo e il diabetologo.

È stato chiesto ai rispondenti di indicare quali siano, nella loro percezione, le principali criticità nella gestione del percorso del pazienti, distinguendo tra arruolamento del paziente e follow-up / rivalutazione.

Le risposte relative alle criticità nella fase di arruolamento mostrano una certa incertezza dei rispondenti (Figura 3): se la lunghezza del periodo di pre-trattamento con statine ad alto dosaggio e ezetimibe è percepita come la principale criticità e l'estensione della prescrivibilità ad altri specialisti come un elemento non prioritario, gli altri aspetti (dalla numerosità dei centri al referral, alla complessità della scheda di eleggibilità, alla necessità di tre esami di laboratorio) sembrano presentare una criticità molto simile nella percezione dei rispondenti. Tale incertezza si riflette nel valore molto basso dell'indice di concordanza (indice $\mathrm{W}$ di Kendall), pari al 10,5\%. È importante osservare come gran parte delle dimensioni sopra rilevate sia trasversale alle regioni, mentre la numerosità dei centri sia conseguenza di scelte differenti a 


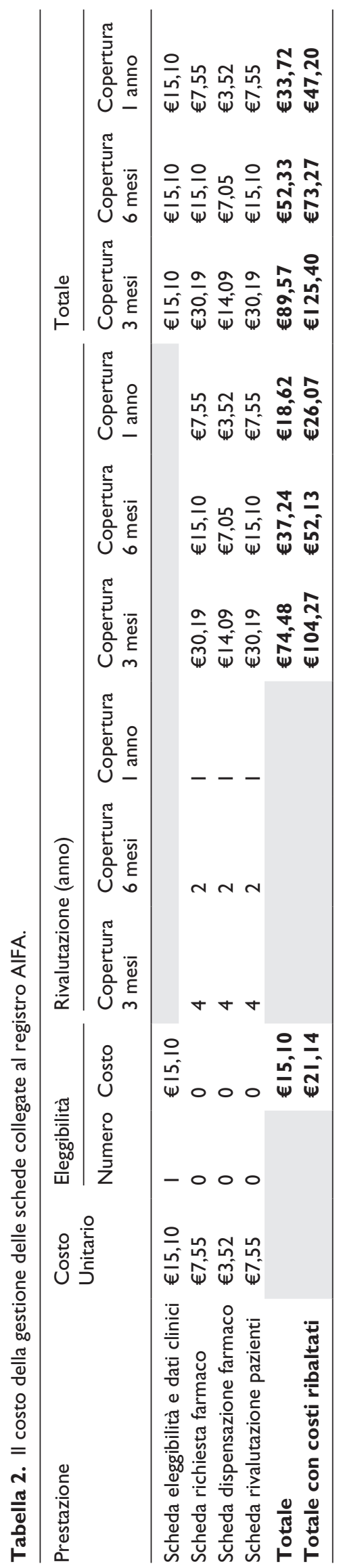

livello regionale e, quindi, è abbastanza naturale che il grado di rilevanza attribuito a tale aspetto sia variabile. Con riferimento alla scelta dei centri prescrittori, i rispondenti hanno sottolineato che non solo i criteri di scelta ma anche le modalità di abilitazione dei centri siano differenti. Ad esempio, è stato segnalato come in Sicilia l'abilitazione sia avvenuta su richiesta dei centri, con conseguente annullamento della stessa in caso di mancato reclutamento dei pazienti entro un determinato periodo di tempo.

Con riferimento al percorso di rivalutazione dei pazienti (Figura 4), il quadro delle valutazioni soggettive è più omogeneo rispetto a quello sull'arruolamento dei pazienti, ma con valori dell'indice di concordanza ancora molto bassi (di poco superiori al 15\%).

La durata di copertura dei registri, la tipologia di specialisti coinvolti e il limitato numero di centri rappresentano le maggiori criticità organizzative, pur essendo la numerosità dei centri un problema sentito in modo abbastanza variabile, date le forti differenze interregionali. La dispensazione in distribuzione diretta risulta, invece, il problema meno rilevante (in linea con l'accordo piuttosto basso sul passaggio alla Distribuzione Per Conto (DPC) - cfr. Tabella 4), nonostante non sempre vi sia allineamento tra rivalutazione e dispensazione del farmaco. Tra le schede da compilare nella fase di follow-up è emersa una maggiore criticità della scheda di dispensazione del farmaco, valutazione espressa anche da alcuni clinici.

La Tabella 4 evidenzia come, in termini generali, il livello di accordo sia più alto su una modifica (o mantenimento) del periodo di copertura del registro a sei mesi, con eventuale opzione di arrivare fino a un anno e su una possibilità di gestire il follow-up del paziente in ambulatori territoriali, pur mantenendo l'arruolamento a livello ospedaliero. Con riferimento al periodo di esposizione al trattamento con statine ad alto dosaggio ed ezetimibe, vi è un accordo a ridurlo a tre mesi, ma non a un mese (su questa ipotesi c'è anche una decisa variabilità nelle opinioni dei rispondenti). Il livello di consenso sulla riduzione del numero di rilevazioni di LDL-C a due, così come su un'eventuale modifica delle modalità distributive dei farmaci da diretta a DPC è intermedio. Nello specifico, è stato sottolineato come sarebbe più opportuno adottare, in previsione della gestione del follow-up sul territorio (cfr. infra), una soluzione intermedia tra distribuzione diretta e DPC, rappresentata dalla distribuzione dei farmaci da parte dei servizi farmaceutici territoriali e/o degli ambulatori di distretto.

I rispondenti hanno fornito spunti sulle azioni da perseguire, sintetizzati in Appendice. Oltre ad alcune indicazioni specifiche sui temi già discussi e una generale richiesta di una maggiore omogeneità delle scelte regionali, è stata sottolineata la limitata conoscenza della materia da parte di una quota consistente della classe medica potenzialmente coinvolta nel percorso di cura dei pazienti 
Tabella 3. Il costo totale della gestione del percorso terapeutico collegato a farmaci PCSK9-inibitori in presenza e assenza di compartecipazioni a carico del paziente.

\begin{tabular}{|c|c|c|c|c|c|}
\hline \multirow{2}{*}{$\begin{array}{l}\text { Pazienti non esenti } \\
\text { da compartecipazioni } \\
\text { alla spesa }\end{array}$} & \multicolumn{2}{|c|}{ Prestazioni ambulatoriali (a carico pazienti) } & \multirow{2}{*}{$\begin{array}{l}\text { Schede farmaci } \\
\text { (a carico SSN) }\end{array}$} & \multicolumn{2}{|l|}{ Totale } \\
\hline & Lordo superticket & Netto superticket & & Lordo superticket & Netto superticket \\
\hline Copertura tre mesi & $€ 27 I, 53$ & $€|5|, 53$ & $€ I 25,40$ & $€ 396,93$ & $€ 276,93$ \\
\hline Copertura sei mesi & $€ I 76,43$ & $€ 96,43$ & $€ 73,27$ & $€ 249,70$ & $€ 169,70$ \\
\hline Copertura un anno & $€ \mid 28,88$ & $€ 68,88$ & $€ 47,20$ & $€ I 76,08$ & $€ I \mid 6,08$ \\
\hline $\begin{array}{l}\text { Pazienti esenti da } \\
\text { compartecipazioni } \\
\text { alla spesa }\end{array}$ & \multicolumn{2}{|c|}{$\begin{array}{l}\text { Prestazioni arnbulatoriali (a carico } \\
\text { SSN) }\end{array}$} & $\begin{array}{l}\text { Schede farrnaci } \\
\text { (a carico SSN) }\end{array}$ & \multicolumn{2}{|l|}{ Totale } \\
\hline Copertura tre mesi & \multicolumn{2}{|l|}{$€ 15 \mid, 53$} & $€ I 25,40$ & \multicolumn{2}{|l|}{$€ 276,93$} \\
\hline Copertura sei mesi & \multicolumn{2}{|l|}{$€ 96,43$} & $€ 73,27$ & \multicolumn{2}{|l|}{$€ 169,70$} \\
\hline Copertura un anno & \multicolumn{2}{|l|}{$€ 68,88$} & $€ 47,20$ & \multicolumn{2}{|l|}{$€|| 6,08$} \\
\hline
\end{tabular}

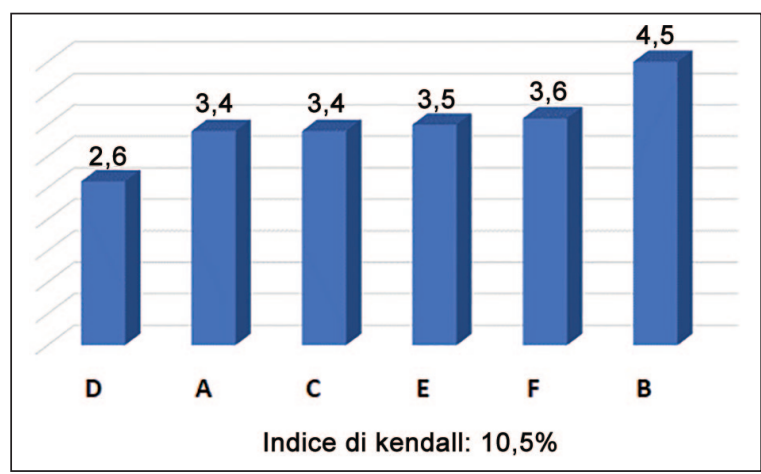

Figura 3. Grado di rilevanza attribuito alle principali criticità in fase di arruolamento del paziente eleggibile a PCSK9-inibitori (punteggio medio*).

* Le proposte sono state classificate attribuendo I alla proposta più importante e 6 a quella meno rilevante. Un punteggio medio-basso è quindi indice di alta priorità.

Legenda

A. Numerosità limitata dei centri.

B. Tipologia limitata degli specialisti.

C. Complessità della scheda di eleggibilità e dati clinici.

D. Lunghezza del periodo di pre-trattamento con statine ad alto dosaggio e ezetimibe

E. Necessità di tre esami di laboratorio per la verifica di non efficacia I intolleranza al trattamento.

F. Referral al centro prescrittore non ottimale.

con ipercolesterolemia, aspetto da considerare a maggior ragione in caso di attribuzione della responsabilità del follow-up agli specialisti territoriali.

In generale, nell'opinione dei rispondenti, l'ipercolesterolemia non sembra essere percepita come un elemento di particolare rilievo clinico da molti medici (e da molti cittadini) che, potenzialmente, possono interagire con pazienti eleggibili ai PCSK9-inibitori. Un elemento chiave è che la semplificazione del percorso, richiamata da diversi rispondenti, venga associata ad una attività di sostegno alle competenze cliniche di base. Particolare rilievo è stato dato all'attività di informazione / formazione del Medico di Medicina Generale (MMG) e alla creazioni di team

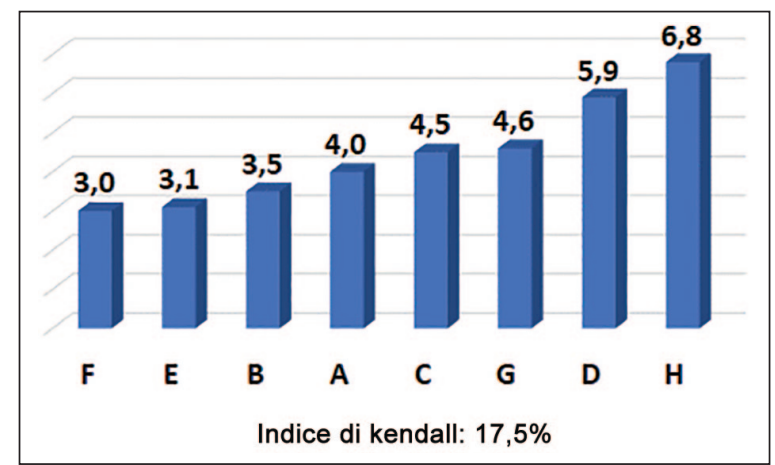

Figura 4. Grado di rilevanza attribuito alle principali criticità nella fase di rivalutazione del paziente eleggibile a PCSK9inibitori (punteggio medio*).

* Le proposte sono state classificate attribuendo I alla proposta più importante e 8 a quella meno rilevante. Un punteggio medio basso è quindi indice di alta priorità.

Legenda

A. Numerosità limitata dei centri.

B. Tipologia limitata degli specialisti.

C. Complessità della scheda di rivalutazione.

D. Complessità della scheda di richiesta farmaco.

E. Complessità della scheda di dispensazione farmaco.

F. Durata limitata della copertura dei registri.

G. Numero di accessi del paziente all'ospedale.

$\mathrm{H}$. Dispensazione ospedaliera in distribuzione diretta.

multidisciplinari per la gestione della prevenzione secondaria e della rivalutazione del paziente. In generale le attività di formazione sono rimaste confinate, secondo gli esperti, a piccoli gruppi di specialisti ospedalieri e universitari. Sono stati però segnalati alcuni approccio più strutturati:

- la Regione Friuli Venezia Giulia ha istituito un gruppo di lavoro multidisciplinare sia per discutere e fornire indicazioni sulla identificazione dei centri prescrittori dei farmaci PCSK9-inibitori, sia per elaborare un documento di indirizzo prescrittivo sui nuovi anticorpi monoclonali per la cura dell'ipercolesterolemia primaria. Sulla base delle 
Tabella 4. Grado di accordo su eventuali modifiche del percorso terapeutico associato a PCSK9-inibitori.

\begin{tabular}{ll}
\hline Tipologia di riforme & Media dei punteggi \\
\hline Centri autorizzati & 2.3 \\
Arruolamento e follow-up anche in ambulatori territoriali & 3.0 \\
Arruolamento in ospedale e follow-up anche in ambulatori territoriali & 2.1 \\
Arruolamento & 2.4 \\
Riduzione del tempo di esposizione a statine ad alto dosaggio + ezetimibe ad un mese & 2.7 \\
Riduzione del tempo di esposizione a statine ad alto dosaggio + ezetimibe a tre mesi & 2.2 \\
Riduzione del numero di rilevazioni di LDL-C due & $26 \%$ \\
Riduzione del tempo di esposizione a statine ad alto dosaggio + ezetimibe ad un mese & 2.5 \\
con due rilevazioni di LDL-C & \\
Riduzione del tempo di esposizione a statine ad alto dosaggio + ezetimibe a tre mesi & $29 \%$ \\
con due rilevazioni LDL-C & 3.2 \\
Rivalutazione & 2.6 \\
Allungamento del periodo di copertura registro a sei mesi & 3.1 \\
Allungamento del periodo di copertura del registro a un anna & $35 \%$ \\
Allungamento del periodo di copertura del registro a sei mesi/un anno & $28 \%$ \\
Distribuzione del farmaco al paziente & 2.6 \\
Distribuzione del farmaco in farmacia attraverso DPC*, salvo la prima dispensazione & 2.3 \\
Distribuzione del farmaco in farmacia attraverso DPC* per tutte le dispensazioni & $21 \%$ \\
\hline
\end{tabular}

Nota: Il punteggio attribuito varia da I (per nulla d'accordo) a 4 (molto d'accordo). Un punteggio medio basso è quindi sintomo di un livello di accordo basso.

CV: Coefficiente di variazione dei punteggi attribuiti; DPC: Distribuzione Per Conto.

proposte formulate dal gruppo di lavoro e di concerto con le direzioni aziendali delle Aziende del Servizio Sanitario Regionale, la Direzione Centrale Salute della Regione ha definito i centri prescrittori. La Regione ha inoltre organizzato un evento sul tema con il coinvolgimento di tutti gli attori (specialisti, MMG, farmacisti) per informare sulle evidenze di letteratura, sui rispettivi ruoli durante la terapia e sul percorso da seguire per la corretta prescrizione e dispensazione dei farmaci PCSK9-inibitori. È stato infine predisposto un documento per valutare l'epidemiologia dell'ipercolesterolemia primaria nel contesto regionale, per analizzare le evidenze di efficacia e sicurezza dei nuovi trattamenti disponibili individuando $i$ pazienti che potrebbero essere eleggibili alla nuova tipologia di farmaci, nonché per esaminare le caratteristiche dei centri prescrittori;

- la Regione Lazio ${ }^{19}$ ha previsto una scheda di referral ai centri prescrittori, compilabile da qualsiasi medico operante nel Servizio Sanitario Regionale, anche se risulta che tale scheda sia scarsamente utilizzata;

- un rispondente della Regione Campania ha citato un'esperienza di collaborazione con Cooperative di MMG che ha comportato un sensibile aumento di referral da parte degli stessi MMG.

Nonostante tali iniziative, rimangono comunque un limitato coinvolgimento dei $\mathrm{MMG}$ nel percorso del paziente, una scarsa (o assente) conoscenza dell'opportunità terapeutica fornita dai PCSK9-Inibitori da parte degli stessi MMG e, ancor più in generale, una sensibilità abbastanza bassa dei pazienti e degli operatori sanitari. Nello specifico, sono stati segnalati tre problemi:

- nella prevenzione primaria il referral delle ipercolesterolemie genetiche non è frequente, quale conseguenza di una scarsa attenzione alla loro diagnosi;

- il percorso di valutazione nella finestra di trattamento di sei mesi è spesso incompleto, con referral non ottimale a centri prescrittori e successivo re-invio del paziente al MMG;

- per i pazienti in prevenzione secondaria risulta poco frequente il monitoraggio dei livelli di LDL-C dopo la dimissione per un evento ischemico acuto.

Più in generale, la percezione del problema dell'ipercolesterolemia sembra essere ancora limitata, considerando che metà della popolazione italiana ha LDL-C $>115 \mathrm{mg} / \mathrm{dl}$ (quindi, in molti casi, suscettibile di trattamento in base alla nota 13 AIFA). Infatti, nel documento di strategia 2017 "Alleanza Italiana per le Malattie Cardio-cerebrovascolari” del Ministero della Salute, ${ }^{20}$ viene evidenziato come il trattamento dell'ipercolesterolemia non sia migliorato rispetto al passato, mentre la consapevolezza su altri fattori di rischio (soprattutto ipertensione, ma anche fumo) e il relativo eventuale trattamento siano aumentati. Risulta quindi necessario, secondo i rispondenti, rafforzare la consapevolezza di soggetti istituzionali e di assistiti di quanto l'ipercolesterolemia sia importante come fattore di 
rischio, di quanto poco sia trattata e dell'impatto della sua cura in termini di guadagno di salute.

\section{Conclusioni}

Il presente articolo contiene le evidenze di uno studio Delphi su 12 panelist (clinici e farmacisti), sulla gestione del percorso del paziente potenzialmente eleggibile al trattamento con farmaci PCSK9-inibitori. Dallo studio sono emersi diversi spunti di riflessione.

In primo luogo, è stato rilevato come l'attuale sistema di gestione del percorso terapeutico dei pazienti candidabili al trattamento con PCSK9-inibitori possa essere migliorato.

Per quanto esista una certa variabilità / incertezza sulle azioni da intraprendere per migliorare tale percorso, emergono tre elementi sui quali esiste un certo grado di accordo, rappresentati, in ordine di rilevanza, da:

- un aumento del periodo di copertura del registro a sei mesi in tutte le realtà, per arrivare eventualmente anche a un anno;

- una gestione della rivalutazione dei pazienti negli ambulatori territoriali, salvo il mantenimento in ambito ospedaliero dell'arruolamento del paziente;

- una riduzione a tre mesi del periodo di trattamento dei pazienti con statine ad alto dosaggio in associazione a ezetimibe per la verifica della mancata risposta / intolleranza a statine e a due degli esami di laboratorio.

Rispetto al tema della distribuzione del farmaco, gli esperti hanno evidenziato l'opportunità di prevedere, dato il follow-up territoriale, il passaggio della distribuzione diretta dall'ambito ospedaliero a quello territoriale, con una minore propensione all'attivazione della DPC. Il periodo di copertura ottimale della terapia fornita al paziente per uso a domicilio sarebbe pari a tre mesi: un allungamento a sei mesi / un anno potrebbe generare una minore aderenza, rischio di conservazione non corretta (dovendo il prodotto essere conservato in frigorifero a $2^{\circ}-8^{\circ}$ ), effetti finanziari per le aziende sanitarie più concentrati nel tempo (l'acquisto avverrebbe infatti una / due volte all'anno, anziché quattro).

I rispondenti hanno comunque evidenziato come sia cruciale il tema dell'effettiva presa in carico del paziente: la loro percezione è che gran parte dei pazienti attualmente in terapia siano quelli già in carico presso le strutture specialistiche abilitate alla prescrizione. Sono quindi considerati fondamentali (i) l'informazione e la formazione del MMG e degli specialisti territoriali, (ii) l'appropriato referral da parte degli stessi alle strutture specialistiche abilitate alla prescrizione, (iii) una corretta gestione, anche in previsione di un follow-up territoriale, della rete dei soggetti coinvolti (referral del MMG, arruolamento di centri ospedalieri e follow-up di centri specialistici ambulatoriali), (iv) una maggiore sensibilizzazione dei pazienti, con un effetto di ritorno positivo non solo sull'accesso dei PCSK9-inibitori ma, più in generale, su una migliore gestione delle terapie ipolipemizzanti disponibili.

Viene poi sottolineata la necessità di rendere maggiormente coordinato il percorso, concentrandosi sugli aspetti che maggiormente contribuiscono all'appropriatezza clinica in fase di reclutamento e follow-up del paziente.

Un ulteriore elemento richiamato dai rispondenti è la necessità di una maggiore omogeneità a livello territoriale, inter e infra regionale. In linea di principio, l'esistenza di un registro dovrebbe ridurre la variabilità, rendendo più omogeneo il processo di arruolamento e follow-up dei pazienti, ma la variabilità consentita sulla durata di copertura del registro rende di fatto non omogenea la gestione del paziente.

In termini generali, le modifiche del percorso, a parità di appropriatezza prescrittiva e di aderenza al trattamento, potrebbero avere un effetto sui costi di gestione dello stesso: una copertura a tre / sei mesi / un anno del registro genera un costo annuo a carico del SSN rispettivamente di $€ 276,93, € 169,70$ e $€ 116,08$, se le prestazioni ambulatoriali fossero a carico del SSN. Nel caso di prestazioni a carico dei pazienti, l'SSN sosterrebbe il solo costo di gestione delle schede di reclutamento, pari rispettivamente a $€ 125,40, € 73,27$ e $€ 47,20$ nei tre scenari, mentre i costi massimi a carico dei pazienti (ovvero in presenza di un superticket oltre alle compartecipazioni ordinarie) sarebbero pari a $€ 271,53, € 176,43$ e $€ 128,88$.

Le risposte dei rispondenti hanno evidenziato una sostanziale coerenza tra criticità e soluzioni prospettate. $\mathrm{Ci}$ sono però alcune discrepanze: ad esempio, la durata del periodo di valutazione del paziente per l'eleggibilità al trattamento è stata percepita come uno degli ostacoli principali, ma è emersa come la terza priorità di intervento. Tale discrepanza è stata discussa con gli esperti nell'incontro finale, ed è emerso come nella lettura degli ostacoli i rispondenti abbiano fatto principalmente riferimento alla propria esperienza, mentre nella valutazione degli interventi prioritari sia prevalsa una visione di sistema (che ha reso molto più rilevante, ad esempio, l'estensione della copertura del registro).

Lo studio Delphi e la discussione dei risultati in plenaria hanno fatto emergere alcune ulteriori riflessioni di interesse. Ad esempio, è risultato come la rilevazione dei costi della gestione delle schede possa indurre a percepire tale attività come uno "spreco di risorse" in quanto costo sostenuto dalle aziende sanitarie e sottratto ad altre attività (cosiddetto costo-opportunità). È stato però ribadito che la compilazione delle schede rappresenta un obbligo regolatorio e non un'attività opzionale: è perciò importante ottimizzare tale attività ai fini del raggiungimento dell'obiettivo di un registro, ossia rendere appropriati l'identificazione del paziente eleggibile al trattamento, la prescrizione dei farmaci e il follow-up del paziente.

La presente analisi, per quanto presenti alcuni limiti (ridotta numerosità dei rispondenti, chiusura del Delphi 
con un incontro in aperto) e non voglia essere rappresentativa dell'opinione / percezione di tutte le realtà sul territorio, fornisce importanti spunti di riflessione per il migliore accesso e gestione dei PCSK9-inibitori, così come, in futuro, di farmaci simili sotto il profilo del percorso prescrittivo e distributivo.

\section{Ringraziamenti}

La presente ricerca è stata svolta in collaborazione con 12 panelist partecipanti allo Studio Delphi e che hanno condiviso il documento finale qui presentato:

- sette clinici: Marcello Arca (Università di Roma Sapienza), Maurizio Averna (Università degli Studi di Palermo), Paolo Calabrò (Università degli Studi della Campania Luigi Vanvitelli), Claudio Ceconi (Azienda Socio-Sanitaria Territoriale del Garda), Furio Colivicchi (ASL Roma 1), Aldo Pietro Maggioni (Centro Studi ANMCO), Pasquale Perrone Filardi (Azienda Ospedaliero-Universitaria Federico II);

- cinque farmacisti: Roberta Di Turi (ASL Roma 3), Stefano Palcic (Azienda Sanitaria Universitaria Integrata di Trieste), Maurizio Pastorello (Azienda Sanitaria Provinciale di Palermo), Alessia Pisterna (Azienda Ospedaliera "Maggiore della Carità" di Novara), Ugo Trama (Regione Campania).

Si ringraziano Dario Lidonnici e Matteo Morichi di MA Provider per il supporto operativo nella gestione dei panelist.

\section{Declaration of conflicting interests}

The authors declared no potential conflicts of interest with respect to the research, authorship and/or publication of this article.

\section{Funding}

The Centre For Research on Health and Social Care Management (CERGAS) - SDA Bocconi received an unconditional grant from Amgen to conduct this research.

\section{ORCID iD}

Claudio Jommi (iD) https://orcid.org/0000-0003-0356-8710

\section{Riferimenti bibliografici}

1. Osservatorio Nazionale sull'impiego dei Medicinali (OsMed). L'uso dei farmaci in Italia. Rapporto Nazionale 2017. Roma: Agenzia Italiana del Farmaco, 2018, http:// www.aifa.gov.it/content/rapporti-osmed-luso-dei-farmaciitalia (ultimo accesso 24 novembre 2018).

2. Regione Veneto. Documento di HTA proposto per un accesso controllato al mercato dei nuovi anticorpi monoclonali per la cura dell'ipercolesterolemia primaria. Allegato A al Decreto n. 98 del 6 ottobre 2016. Venezia: Regione Veneto, Gruppo di Lavoro sui Farmaci per la cura dell'ipercolesterolemia e della dislipidemia, 2016.

3. Martini N, Arca M, Averna M, et al. Concept paper sul Progetto inibitori di PCSK9: accesso e sostenibilità. I Supplementi di Politiche Sanitarie 2019; 1: 1-40.

4. Agenzia Italiana del Farmaco (AIFA). Determina 1 febbraio 2017. Classificazione del medicinale per uso umano "Repatha" ai sensi dell'art. 8, comma 10, della legge 24 dicembre 1993, n. 537. (Determina n. 172/2017). (17A01047) (GU Serie Generale n. 31 del 07-02-2017).

5. Agenzia Italiana del Farmaco (AIFA). Determina 16 febbraio 2017. Classificazione del medicinale per uso umano "Praluent" ai sensi dell'art. 8, comma 10, della legge 24 dicembre 1993, n. 537. (Determina n. 256/2017). (17A01583) (GU Serie Generale n. 54 del 06-03-2017).

6. Suh DC, Griggs SK, Henderson ER, et al. Comparative effectiveness of lipid-lowering treatments to reduce cardiovascular disease. Expert Rev Pharmacoecon Outcomes Res 2018; 18 (1): 51-69.

7. Hlatky MA e Kazi DS. PCSK9 Inhibitors: Economics and Policy. J Am Coll Cardiol 2017; 70 (21): 2677-2687.

8. Korman MJ, Retterstøl K, Kristiansen IS, et al. Are PCSK9 Inhibitors Cost Effective? Pharmacoeconomics 2018; 36(9): 1031-1041.

9. Kendall M e Gibbons JD. Rank Correlation Methods. 5th Edition. London: Edward Arnold, 1990.

10. Joshi A, Kale S, Chandel S, et al. Likert Scale: Explored and Explained. Br J Appl Sci Technol 2015; 7 (4): 396-403.

11. Esmé G, Trevelyan J and Robinson C. Delphi methodology in health research: how to do it? Eur J Integr Med 2015; 7(4): c423-428.

12. Lohr S. Sampling: Design and Analysis. 2nd ed. Boston, MA: Brooks/Cole, 2010.

13. Ministero della Salute. Nomenclatore tariffario dell'assistenza specialistica ambulatoriale (Decreto del Ministero della Salute 18 ottobre 2012), http://www.salute .gov.it (ultimo accesso 24 giugno 2018).

14. Armeni A, Bertolani A, Borsoi L, et al. La spesa sanitaria: composizione ed evoluzione. In: CERGAS - Bocconi (a cura di) Rapporto OASI 2018. Milano: Egea S.p.A., 2018.

15. Ciancaleoni Bartoli I. Che cos'è l'Ipercolesterolemia Familiare. OMAR - Osservatorio Malattierare, http://www .osservatoriomalattierare.it/index.php/ipercolesterolemia -familiare/1199-che-cose-lipercolesterolemia-familiare (ultimo accesso 24 giugno 2019).

16. Farmacisti al Lavoro. Lo stipendio del farmacista ospedaliero, https://www.farmacistiallavoro.it/2017/03/06 /lo-stipendio-del-farmacista-ospedaliero/ (ultimo accesso 24 giugno 2019).

17. Agenzia per la Rappresentanza Negoziale delle Pubbliche Amministrazioni (ARAN). Retribuzioni medie PA per macro voce, https://www.aranagenzia.it/ (ultimo accesso 24 giugno 2019).

18. Cortese B, Di Palma G, Lettieri C, et al. Inibitori di PCSK9: il difficile connubio fra evidenze scientifiche e limitazioni regolatorie. G Ital Cardiol 2018; 19(2): 77-80.

19. Regione Lazio. Determinazione G10108 del 18/7/2017: Recepimento delle "Linee di indirizzo per il trattamento della ipercolesterolemia" ai sensi delle Determine AIFA di autorizzazione dei farmaci evolocumab e alirocumab, http:// www.regione.lazio.it/binary/rl_farmaci/tbl monitoraggio AIFA/Determinazione G 10108 de $\bar{l}$ 17.7.2018 _PRALUENT_REPATHA.pdf (ultimo accesso 24 giugno 2019).

20. Ministero della Salute. Alleanza Italiana per le Malattie Cardio-cerebrovascolari - Documento di strategia, 2017, http://www.salute.gov.it/portale/documentazione/p6 2_2_1.jsp?lingua $=$ italiano\&id $=2606$ (ultimo accesso 24 giugno 2019). 


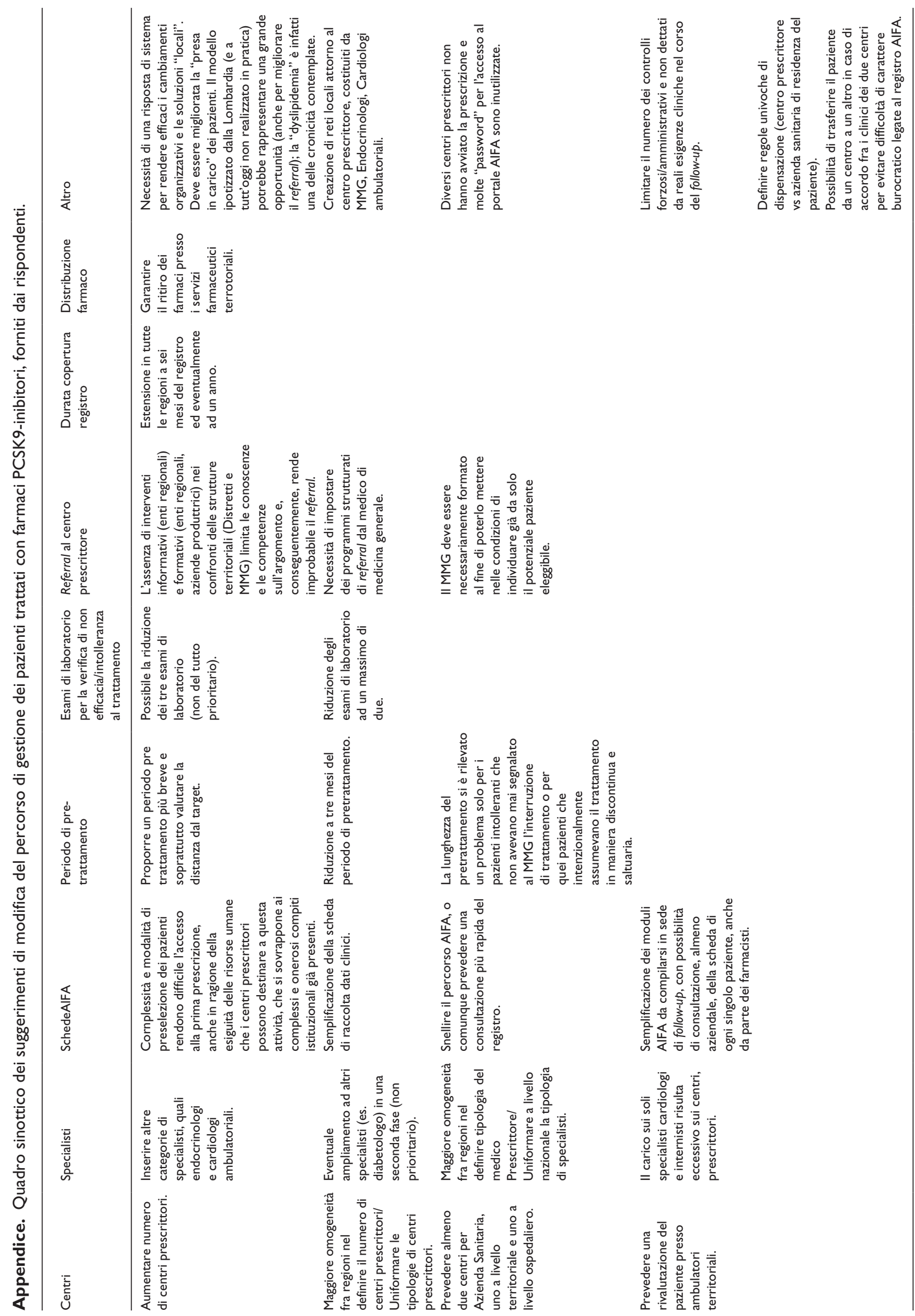

